\title{
Sars-CoV-2/Covid-19 em Pacientes com Câncer
}

https://doi.org/10.32635/2176-9745.RBC.2020v66n2.970

SARS-CoV-2/COVID-19 in Patients with Cancer
Sars-CoV-2/Covid-19 en Pacientes con Cáncer

Luiz Claudio Santos Thuler'; Andreia Cristina de Melo²

Em 12 de dezembro de 2019, um conjunto de casos de pneumonia causada por um coronavírus recém-identificado foi anunciado em Wuhan, China. Esse coronavírus foi inicialmente nomeado como novo coronavírus de 2019 (2019nCoV) em 12 de janeiro de 2020 pela Organização Mundial da Saúde (OMS). Rapidamente, instalou-se uma epidemia de infecção aguda do trato respiratório, tendo a OMS nomeado oficialmente a doença como doença pelo coronavírus 2019 (coronavirus disease 2019 - Covid-19), e o novo coronavírus como o vírus da síndrome respiratória aguda grave do coronavírus 2 (severe acute respiratory syndrome coronavirus 2 - Sars-CoV-2) ${ }^{1}$.

Como uma doença infecciosa respiratória aguda emergente, a Covid-19 se espalha principalmente pelo trato respiratório, por gotículas, secreçóes respiratórias e contato direto ${ }^{2}$. Entretanto, há relatos de que o Sars-CoV-2 tenha sido isolado de swabs fecais e de sangue, indicando a possibilidade de transmissáo por múltiplas rotas ${ }^{1}$.

A doença é altamente transmissível entre seres humanos. Os pacientes apresentaram sintomas como febre, mal-estar e tosse $e^{3}$ Embora o sinal clínico inicial da Covid-19, que permitiu a detecção de casos, tenha sido a pneumonia, há relatos mais recentes de sintomas gastrointestinais e infecçōes assintomáticas, principalmente em crianças .

Os fatores de risco para evolução com quadro clínico grave e morte por infecção por Covid-19 incluem idade avançada e presença de comorbidades, características comuns em pacientes com câncer ${ }^{5}$. Além disso, o próprio câncer, juntamente com seu tratamento, torna os pacientes com neoplasias mais suscetíveis a pneumonias, em razão da resposta imunológica enfraquecida por bactérias e vírus respiratórios. Assim, a disseminação epidêmica do Sars-CoV-2 traz grandes desafios para a prática clínica nas áreas da oncologia e da hematologia ${ }^{6}$.

Apesar do número de artigos científicos disponíveis no PubMed sobre a Sars-CoV-2/Covid-19 passar de três mil (em 7 de abril de 2020), há poucos estudos clínicos consistentes sobre o risco aumentado de infecção em pacientes com câncer e seu impacto no prognóstico.

Em um relato pioneiro de Liang et al. ${ }^{7}$, publicado no The Lancet Oncology, de 1.590 casos com Covid-19 confirmada, 18 pacientes tinham histórico de câncer (prevalência $=1,1 \%$ ). Comparados a pacientes sem câncer, os pacientes com câncer eram mais idosos (média de idade de 63,1 anos vs 48,7 anos), apresentavam mais frequentemente história de tabagismo (22\% vs 7\%), tiveram mais polipneia ( $47 \%$ vs $23 \%)$ e manifestação inicial mais grave visibilizada na tomografia computadorizada de tórax ( $94 \%$ vs $71 \%)$, mas não apresentaram diferenças significantes quanto ao gênero, a sintomas basais, e a outras comorbidades ou gravidade basal na radiografia de tórax. Os autores concluíram que os pacientes com câncer apresentavam maior risco de apresentar Covid-19 e com pior prognóstico do que aqueles sem câncer. Este relato tem sido apontado como o primeiro a focar na ocorrência da Covid-19 em pacientes com câncer ${ }^{8}$. Entretanto, Xia et al. ${ }^{9}$ destacaram que foi estudado um número pequeno de casos, com diversos tipos de câncer de comportamentos biológicos diferentes, cursos de doenças altamente variáveis (de 0 a 16 anos) e estratégias de tratamento diversas, o que pode não ser representativo da população com câncer. Além disso, cabe destacar que a média de idade desses pacientes (63,1 anos) era significantemente maior do que naqueles sem câncer (48,7 anos), sugerindo que a idade avançada estaria associada à pior evolução da Covid-19. Outro aspecto é que a proporção de fumantes era muito maior nos pacientes com câncer, sobretudo nos casos de câncer de pulmão, o que tem sido apontado como fator de gravidade da doença.

Em outro estudo, Zhang et al. ${ }^{10}$ identificaram retrospectivamente 28 pacientes com câncer entre 1.276 pacientes com Covid-19 (prevalência $=2,2 \%$ ) internados em três hospitais em Wuhan, China, entre janeiro e fevereiro de 2020. Os autores forneceram a primeira estimativa da probabilidade de morte em pacientes com câncer e Covid-19,

${ }^{1}$ Divisão de Pesquisa Clínica e Desenvolvimento Tecnológico do Instituto Nacional de Câncer José Alencar Gomes da Silva (INCA). Rio de Janeiro (RJ), Brasil. Orcid iD: https://orcid.org/0000-0003-2550-6537.E-mail: Ithuler@inca.gov.br

2 Divisão de Pesquisa Clínica e Desenvolvimento Tecnológico do INCA. Rio de Janeiro (RJ), Brasil. Orcid iD: https://orcid.org/0000-0002-1201-4333.

E-mail: andreia.melo@inca.gov.br 
apresentando taxa de letalidade de $28,6 \%$, cerca de dez vezes superior à relatada no conjunto de pacientes com Covid-19 da China. Oh ${ }^{5}$ comenta o estudo e destaca que se deve ter cautela na interpretação desses resultados por se tratar de uma série pequena de pacientes, limitada aos casos mais graves, com dados coletados retrospectivamente, tornando a extrapolação para outros países problemática. Conclui sugerindo que, apesar disso, pacientes com câncer devem, no mínimo, praticar distanciamento ou isolamento social e serem candidatos à avaliação clínica rápida e precoce frente a sintomas suspeitos de Covid-19, incluindo testes virais e exames de imagem de tórax.

Em recente metanálise ${ }^{11}$, que incluiu artigos publicados até 14 de março de 2020 utilizando os termos "COVID-19", "novel coronavirus", "SARS-CoV-2", "2019-nCov", e "cancer", "neoplasm”, "tumor", "malignancy”, foram identificados 276 artigos, dos quais, 266 foram excluídos por duplicação. A análise dos 11 artigos selecionados mostrou que a prevalência de câncer em pacientes com Covid-19 é de 2,0\% (IC95\% 2,0\% - 3,0\%), sugerindo que pacientes e sobreviventes de câncer são uma importante população de risco para a Covid-19.

Diante desses relatos pioneiros, pode-se concluir que, aparentemente, pacientes com câncer apresentam risco mais elevado do que a população geral de desenvolver a Covid-19, evoluindo com elevadas taxas de letalidade. Entretanto, são inúmeros os desafios no que diz respeito à prevenção e ao controle da Sars-CoV-2/Covid-19, havendo ainda muitas questôes a serem respondidas pela ciência.

\section{REFERÊNCIAS}

1. Guo YR, Cao QD, Hong ZS, et al. The origin, transmission and clinical therapies on coronavirus disease 2019 (COVID-19) outbreak - an update on the status. Mil Med Res. 2020;7(1):11. doi: https://doi.org/10.1186/s40779020-00240-0

2. Li Q, Guan X, Wu P, et al. Early transmission dynamics in Wuhan, China, of novel coronavirus-infected pneumonia. N Engl J Med. 2020;382:1199-1207. doi: https://doi.org/10.1056/NEJMoa2001316

3. Huang C, Wang Y, Li X, et al. Clinical features of patients infected with 2019 novel coronavirus in Wuhan, China. Lancet. 2020;395(10223):497-506. doi: https://doi.org/10.1016/S0140-6736(20)30183-5

4. Chan JFW, Yuan S, Kok KH, et al. A familial cluster of pneumonia associated with the 2019 novel coronavirus indicating person-to-person transmission: a study of a family cluster. Lancet 2020;395(10223):514-523. doi: https:// doi.org/10.1016/S0140-6736(20)30154-9

5. Oh WK. COVID-19 infection in cancer patients: early observations and unanswered questions. Ann Oncol. 2020;pii:S0923-7534(20)36384-5. doi: https://doi.org/10.1016/j.annonc.2020.03.297

6. Yang G, Zhang H, Yang Y. Challenges and countermeasures of integrative cancer therapy in the epidemic of COVID-19. Integr Cancer Ther. 2020;19:1534735420912811. doi: https://doi.org/10.1177/1534735420912811

7. Liang W, Guan W, Chen R, et al. Cancer patients in SARS-CoV-2 infection: a nationwide analysis in China. Lancet Oncol. 2020;21(3):P335-337. doi: http://doi.org/10.1016/S1470-2045(20)30096-6

8. Wang H, Zhang L. Risk of COVID-19 for patients with cancer. Lancet Oncol. 2020;21(4):PE181. doi: http://doi. org/10.1016/S1470-2045(20)30149-2

9. Xia Y, Jin R, Zhao J, et al. Risk of COVID-19 for patientswith cancer. Lancet Oncol. 2020;21(4):PE180. doi: http:// doi.org/10.1016/S1470-2045(20)30150-9

10. Zhang L, Zhu F, Xie L, et al. Clinical characteristics of COVID-19-infected cancer patients: a retrospective case study in three hospitals within Wuhan, China. Ann Oncol. 2020;pii:S0923-7534(20)36383-3. doi: http://doi.org/10.1016/j. annonc.2020.03.296

11. Desai A, Sachdeva S, Parekh T, et al. COVID-19 and cancer: lessons from a pooled meta-analysis. JCO Glob Oncol. 2020;6:557-559. doi: http://doi.org/10.1200/GO.20.00097

\section{A REVISTA BRASILEIRA DE CANCEROLOGIA ESTÁ RECEBENDO A SUBMISSÁO DE MANUSCRITOS SOBRE CÂNCER E COVID-19.}

